\title{
Differentiation of Human Medial Prefrontal Cortex Activity Underlies Long-Term Resistance to Forgetting in Memory
}

\author{
Youssef Ezzyat, ${ }^{1}$ Marika C. Inhoff, ${ }^{2}$ and Lila Davachi ${ }^{3}$ \\ ${ }^{1}$ Department of Psychology, University of Pennsylvania, Philadelphia, Pennsylvania 19104, ${ }^{2}$ Department of Psychology, University of California, Davis, \\ California 95616, and ${ }^{3}$ Department of Psychology, Columbia University, New York, New York 10027
}

It is well known that distributing study events over time leads to better memory over long time scales, compared with massing study events together. One explanation for such long-term resistance to forgetting is that distributed study leads to neural differentiation in memory, which supports retrieval of past experiences by disambiguating highly similar memory representations. Neuroanatomical models of episodic memory retrieval propose that the hippocampus and medial prefrontal cortex (MPFC) work together to enable retrieval of behaviorally appropriate memories. However, it is not known how representations in these regions jointly support resistance to forgetting long after initial learning. Using fMRI, we measured differentiation in retrieved memory representations following an extended delay in male and female human participants. After 1 week, word- object associations were better remembered if studied across $2 \mathrm{~d}$ (overnight), allowing associations to be learned in distinct temporal contexts, compared with learning within a single day (same day). MPFC retrieval patterns showed differentiation for overnight relative to same day memories, whereas hippocampal patterns reflected associative retrieval success. Overnight memory differentiation in MPFC was higher for associative than item memories and higher than differentiation assessed over a brain-wide set of retrieval-active voxels. The memory-related difference in MPFC pattern differentiation correlated with memory success for overnight learning and with hippocampal-MPFC functional connectivity. These results show that learning information across days leads to differentiated MPFC memory representations, reducing forgetting after 1 week, and suggest this arises from persistent interactions between MPFC and hippocampus.

Key words: consolidation; episodic memory retrieval; hippocampus; medial prefrontal cortex; pattern separation; temporal context

Significance Statement

Neural activity in both the hippocampus and medial prefrontal cortex (MPFC) has been linked to memory-related representations, but prior work has not examined how these representations support episodic memory retrieval over extended time scales that are characteristic of everyday retrieval. We show that differentiation in MPFC activity 1 week after encoding is higher for retrieved information learned across $2 \mathrm{~d}$ compared with within a single day. In hippocampus, differentiation was greater for detailed memory retrieval but was not influenced by whether information had been learned over 1 or $2 \mathrm{~d}$. Differentiation in MPFC predicted behavioral robustness to forgetting and was correlated with hippocampal-MPFC connectivity. The results suggest that contextbased differentiation supports robust long-term memory via persistent MPFC-hippocampal interactions.

\section{Introduction}

Humans have the ability to retrieve episodic memories long after the initial experience, but not all memories maintain their fidelity over time. Behavioral and computational modeling work (Estes, 1955; Howard and Kahana, 2002; Polyn et al., 2009a,b; Heusser et

\footnotetext{
Received Aug. 13, 2017; revised June 12, 2018; accepted June 12, 2018.

Author contributions: Y.E., M.C.I., and L.D. designed research; Y.E. and M.C.I. performed research; Y.E. and M.C.I. analyzed data; Y.E. and L.D. wrote the paper.

This work was supported by DART Neuroscience and NIH Grant R01 MH074692 to L.D.

Correspondence should be addressed to Dr. Lila Davachi, Department of Psychology, Columbia University,

Schermerhorn Hall, 1190 Amsterdam Avenue \#406, New York, NY 10027. E-mail: Id24@columbia.edu.

https://doi.org/10.1523/JNEUROSCI.2290-17.2018

Copyright $\odot 2018$ the authors $\quad 0270-6474 / 18 / 3810244-11 \$ 15.00 / 0$
}

al., 2016) suggests that context-based differentiation at encoding facilitates later memory retrieval. Memory in these studies is typically assessed following a relatively short delay, leaving open the question of how context-based differentiation at encoding might support retrieval over the longer time scales characteristic of everyday episodic retrieval.

Activity in both the hippocampus and medial prefrontal cortex (MPFC) during encoding has been shown to represent contextual information that supports memory, making each a candidate region for context representation following long delays (Kitamura et al., 2017). In rodents, ensemble activity in hippocampus becomes more differentiated over time in a way that predicts temporal memory for learned information (Manns et al., 
2007; Mankin et al., 2012). Similarly, ensembles in MPFC show pattern differentiation with changes in context, although these representations appear to integrate a broader range of contextual changes (Hyman et al., 2012). In humans, blood oxygenation level-dependent (BOLD) fMRI encoding patterns in hippocampus (DuBrow and Davachi, 2014; Ezzyat and Davachi, 2014; Hsieh et al., 2014) and MPFC (Jenkins and Ranganath, 2016; Tompary and Davachi, 2017) reflect the temporal context of learned information and predict temporal memory. Hippocampal activity also reflects contextual retrieval when memory is tested within $1 \mathrm{~d}$ of learning (Ritchey et al., 2015; Long et al., 2017).

Although activity in both the hippocampus and MPFC reflects memory-related context representations, less is known about how they support memory over delays that extend beyond $1 \mathrm{~d}$. There is growing evidence that interactions between the hippocampus and MPFC are critical to memory retrieval. These interactions occur during and after learning of new information (Siapas et al., 2005; Schlichting and Preston 2016; Guise and Shapiro, 2017; Tompary and Davachi 2017) and are thought to reflect processes in which new hippocampal memory representations are integrated into established MPFC cortical networks. This implies that the hippocampal and MPFC contextual representations that support memory should change over time, with MPFC patterns assuming a more important role in supporting detailed memory retrieval over time. Whereas there is evidence that univariate MPFC fMRI activity is greater during retrieval of temporally remote compared with recent memories (Takashima et al., 2006; Gais et al., 2007; Sterpenich et al., 2009; Nieuwenhuis and Takashima, 2011) and more differentiable for remote compared with recent memories (Bonnici et al., 2012), there is little evidence that MPFC patterns reflect contextual information for memories that are all equally remote.

Here, we ask how learning information across contexts leads to neural pattern differentiation in MPFC and hippocampus that supports resistance to forgetting. Each participant was trained to a high criterion (90\%) on two separate lists of word-object associations. To manipulate whether associations were learned in the same or distinct contexts, training sessions occurred either across a $24 \mathrm{~h}$ period (overnight) or within the same session (same day). One week later, participants were scanned using fMRI during cued retrieval to compare the multivariate patterns of retrieved memories that had been learned in the overnight and same day conditions. To foreshadow the core findings, we observed that learning across a $24 \mathrm{~h}$ period led to more differentiated long-term memory representations in MPFC, which correlated with the behavioral benefit of overnight learning over same day learning. Functional connectivity between MPFC and hippocampus also correlated with the degree of MFPC differentiation, suggesting that context-based differentiation supports memory through hippocampal-cortical interactions at least 1 week after learning.

\section{Materials and Methods}

Participants

Twenty-two healthy volunteers ( 15 female; mean age, 23.3 years; range, 19-29 years) from the New York University and New York City communities participated in this study for payment. All participants were native English speakers with normal or corrected-to-normal vision. Informed consent for this experiment was obtained in a manner approved by the University Committee on Activities Involving Human Subjects at New York University. Three participants were excluded from analysis because of mechanical issues with the fMRI scanner, and all remaining 19 participants were included in behavioral analyses. For the fMRI analyses, two additional participants were excluded because of having fewer than five responses in at least one memory condition.

\section{Stimuli}

The words used in the experiment were taken from a set of 506 adjectives downloaded from the MRC Psycholinguistics Database (http://websites. psychology.uwa.edu.au/school/MRCDatabase/uwa_mrc.htm). For each participant, 72 words were randomly chosen from the pool to be used in the overnight, same day, and single session study conditions; an additional 72 words were randomly chosen for each participant to be used as new lures for the Day 7 retrieval test. Within each of the overnight, same day, and single session study lists, half of the words were paired with pictures of natural objects (e.g., animals, plants) and half were paired with pictures of manmade objects (e.g., tools, kitchen gadgets). All object stimuli were taken from an Internet image search. Our randomization procedure ensured that the assignment of words and images to each other and to the overnight/same day/single session conditions was equally likely for all stimuli.

\section{Experimental design and statistical analysis}

General procedure. On Day 1, participants came into the laboratory and were trained to criterion ( $90 \%$ correct) on a set of word-object pairings (overnight pairs). Twenty-four hours later (Day 2), participants returned to the laboratory and were trained to criterion on a novel set of wordobject pairings (same day pairs). After this training on the same day pairs, participants were retrained to criterion (90\% criterion; for details on training see below, Behavioral procedures) on both sets from Day 1 and Day 2 (Fig. 1A). Thus, participants were trained to criterion twice on both lists; for the overnight list, this occurred across $2 \mathrm{~d}$, and for the same day list, this occurred within the same day. One week later (Day 7), participants came into the laboratory and were trained to criterion on a final novel set of word-object pairings (single session pairs). Immediately after this final training session, participants were brought to the fMRI scanner and were scanned while performing a retrieval test for all the word-object pairings learned across all $3 \mathrm{~d}(\sim 15 \mathrm{~min}$ delay between the final study session in the laboratory and the start of the scanned retrieval test). The results of this retrieval test were analyzed to look for behavioral evidence of differences in memory performance on the basis of study condition (overnight/same day/single session), as well as neural evidence for differentiation in memory representations on the basis of study condition when controlling for memory.

Behavioral procedures. On Day 1, participants came into the laboratory and were trained to learn 72 word-object associations (overnight pairs). Training began with a study phase in which participants were presented with all of the word-object pairs and asked to memorize the associations (Fig. 1B, left; no behavioral response was required). Each study phase trial began with $500 \mathrm{~ms}$ of fixation, followed by presentation of the wordobject pair for $4500 \mathrm{~ms}$. After the study phase, participants began the training phase in which the words were presented alone, and participants had to indicate whether the word had been paired with a "natural" or "manmade" object during study or whether they were unsure (Fig. $1 B$, middle). Each training trial began with $500 \mathrm{~ms}$ of fixation, followed by the presentation of a word for a maximum of $12 \mathrm{~s}$. Once a participant made a natural/manmade choice, feedback was given and the object stimulus was presented alongside the word for $8.5 \mathrm{~s}$. Training was broken into three blocks of 24 trials based on pilot data suggesting faster learning for several smaller blocks compared with a single large block. Once all 24 trials were tested, training began again. If a participant correctly answered the natural/manmade decision on two consecutive training rounds, the pair was dropped from future training rounds. Participants continued with training until they reached $90 \%$ of the 24 pairs correct. The training procedure was then repeated for the second and third blocks of 24 pairs. After reaching the $90 \%$ criterion on the third and final block, participants performed a final test on all 72 pairs together and were required to reach $90 \%$ correct. This was done to ensure that participants still showed robust learning for pairs that had been learned in the first and second blocks. Three participants that performed slightly below the $90 \%$ criterion (minimum, $86 \%$ ) on this final test were re-exposed to the full set of 72 pairs one final time. Participants were then dismissed until the following day. 
A Day 1
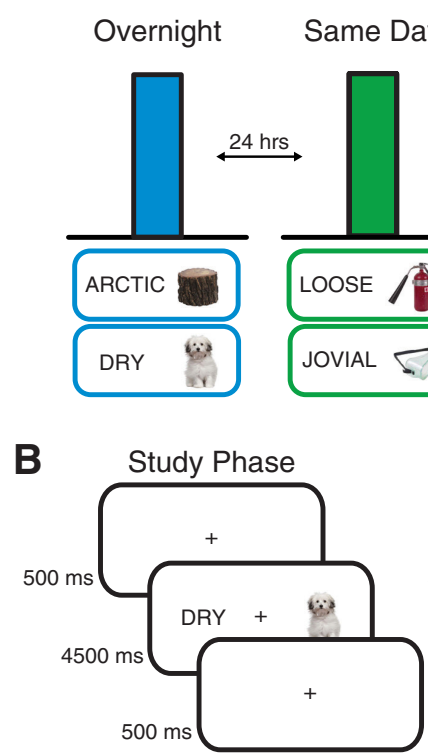

Day 2

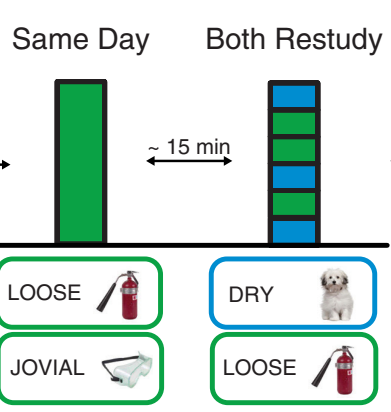

Day 7

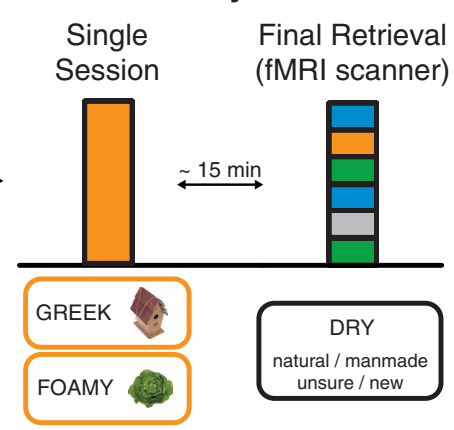

Figure 1. Study design. A, General procedures for the experiment. Day 1: Participants were trained to criterion on a set of word-object associations on Day 1 (overnight condition). Day 2: Twenty-four hours, later participants returned to the laboratory and were trained on a new set of word- object associations (same day condition) before being retrained to criterion on both the overnight and same day lists intermixed. Day 7: One week after the Day 1 session, participants were trained to criterion on a novel set of word-object associations (single session) before being placed in the fMRI scanner and performing a retrieval test that included all lists (overnight, same day and single session) and novel lures. $\boldsymbol{B}$, Left, Each training session began with a study phase in which participants were exposed to the word- object associations. Middle, Training then began with a test in which word- object association memory was tested by having participants respond "natural" or "manmade" for the object that had been associated with each word (Nat, natural; Man, manmade; Uns, unsure). After each response, feedback ("correct"/"incorrect") was given along with presentation of the associated object. A correct response was required in two consecutive training rounds in order for the word- object pair to be dropped from future training. This training protocol was performed for three blocks of 24 stimuli until all 72 pairs from the list were learned. After the final block of 24 , all 72 words were then re-presented for a final test to ensure that participants were above $90 \%$ across the entire list. Participants who did not meet the $90 \%$ criterion on this final test were re-exposed to all 72 pairs in a final study phase. Right, In the scanner participants performed the final retrieval test for all of the words from the overnight, same day and single session lists intermixed with novel lures.

Participants returned 24 h later on Day 2 and were trained to $90 \%$ criterion on a novel set of 72 word-object pairs (same day pairs) using a training paradigm that was identically structured to the training on Day 1. After performing the final test on this new set of pairs, participants performed another training session that included all 72 pairs from Day 1 intermixed with all 72 pairs from Day 2 (Fig. $1 A$ ) in a single block. Thus, participants were retrained to $90 \%$ criterion on both the overnight and same day lists; the critical difference is that overnight pairs were first trained on the previous day, whereas the same day pairs were first trained earlier in that same session. All participants met or exceeded $90 \%$ correct for both overnight and same day lists, after which they were dismissed until the following week.

One week after the Day 1 session, participants returned to the laboratory for the Day 7 session. They began this session with another learning phase on a novel set of 72 word-object pairs (single session pairs). After reaching criterion on this set, participants were taken to the fMRI scanner for the final testing session. In the scanner, participants were presented with all of the studied words (72 each of overnight, same day, and single session) as well as 72 novel words (Fig. $1 B$, right) in a randomly ordered sequence. On each trial, participants were presented with a word and were asked to indicate which type of object the word had been paired with during the learning phases (Old-Natural, Old-Manmade, OldUnsure, New). Participants were given a maximum of $6 \mathrm{~s}$ to make their response. After a response, participants performed an odd/even judgment on a series of numbers for the remainder of the trial, up to a total trial length of $24 \mathrm{~s}$.

Behavioral analysis. Analysis of the behavioral data focused on the Day 7 retrieval test and was designed to identify memory differences relating to study list condition (overnight/same day/single session). Trials for which participants responded correctly to the natural/manmade decision (e.g., selecting Old-Natural when the word was old and had been paired with a natural object) were coded as associative; trials for which participants responded incorrectly to the natural/manmade decision (e.g., selecting Old-Natural when the word was old but had been paired with a manmade object) or responded Old-Unsure were coded as item-only. Trials for which participants responded New when the word was "old" were coded as forgotten. Correctly identified new words were coded as correct rejections, and new words that were given any old response were coded as false alarms. Overall memory performance was defined as the difference between the hit rate (any old response to an old trial) minus the false alarm rate. We defined the overnight learning benefit as (overnight associative) (same day associative) proportion correct.

fMRI procedures. As described above in Behavioral procedures, on Day 7 participants first completed the learning phase for the single session stimuli in the laboratory. After completion, participants were brought to the scanner and performed a scanned retrieval test that included all of the learned words (overnight, same day, and single session) as well as novel words (response options: Old-Natural, Old-Manmade, Old-Unsure, New). Participants performed a total of 288 retrieval trials split evenly into 12 scanning runs.

fMRI data acquisition. Functional imaging was performed using a Siemens Allegra 3T head-only scanner with a custom head coil (NM-011; Nova Medical) located at the Center for Brain Imaging at New York University. Functional data were collected using an echoplanar imaging (EPI) pulse sequence ( 34 contiguous slices; TR, $2000 \mathrm{~ms} ; 3 \mathrm{~mm}$ isotropic voxels; TE, $15 \mathrm{~ms}$; flip angle, $82^{\circ}$; field of view, $240 \times 192 \mathrm{~mm}$; slice gap, $0 \%$ ) with slices oriented parallel to the anterior commissure-posterior commissure axis. Slices were positioned ventrally to provide full coverage of the anterior temporal lobes and prefrontal cortex; this resulted in omission of parts of the superior parietal cortex and, occasionally, parts of motor cortex. A high-resolution T1-weighted anatomical scan (magnetization-prepared rapid-acquisition gradient echo sequence, $1 \times$ 
$1 \times 1 \mathrm{~mm}$ ) was also obtained for each subject after the final block of the localizer task.

Preprocessing of $\mathrm{fMRI}$ data. Images were preprocessed using SPM8 software (Wellcome Trust Centre for Neuroimaging). Functional images were realigned to the within-run mean to correct for head motion (one run from one participant was discarded because of head motion $>1$ voxel). Realigned images were corrected for slice acquisition time and were then coregistered to the anatomical image to correct for betweenrun motion. For group-level analyses, the coregistered images were first spatially normalized to an EPI template in Montreal Neurological Institute space, resliced to $2 \times 2 \times 2 \mathrm{~mm}$ voxels, and finally smoothed with a $6 \mathrm{~mm}$ FWHM isotropic Gaussian kernel. Low frequencies ( $<1.5$ cycles/ run) were removed from the functional data in both the subject-specific and group analyses.

\section{ROI definition}

Anatomical hippocampal ROIs were drawn manually on each participant's T1-weighted anatomical scan using an in-house drawing tool written in Matlab (Mathworks) and according to standard anatomical convention (Insausti et al., 1998). For the analysis comparing pattern differentiation in anterior and posterior hippocampus, we split the hippocampus into thirds based on coronal slice position, taking the anterior and posterior thirds as the ROIs. We also defined functional ROIs based on fMRI activation during the retrieval task. We first created an acrossparticipant random-effects general linear model (GLM) that included eight conditions of interest (associative/item-only for each of the overnight/same day/single session lists and correct rejections/false alarms) that modeled activation for each trial as a 2-TR boxcar convolved with a canonical hemodynamic response function (HRF). Trials without a response and forgotten trials were each separately modeled as confounds, as were estimates of across-run participant motion. The resulting $\beta$ estimates were then used to define MPFC; specifically, a contrast of single session associative and item-only trials was conducted against baseline at a voxel-wise threshold of $p<0.001$ to functionally identify the region (MNI coordinates: $0,56,18$ ). Based on a comparison of our region to available MNI coordinates of prior work, the center of our MPFC region is more anterior and/or dorsal to some ROIs that have been reported previously (Gais et al., 2007; Takashima et al., 2007; Bonnici et al., 2012) but is partially overlapping with others (Tompary and Davachi, 2017). Importantly, we used only single session trials to define the MPFC region to avoid biasing our main pattern similarity analyses, which were limited to comparisons between the overnight and same day conditions.

We also defined, individually for each subject, a mask of the 5000 voxels showing the strongest responses (positive or negative) in a contrast of Task $>$ Baseline. We defined these brain-wide task active masks as a proxy for voxels that might be expected to show reinstatement of contextual information during retrieval (Howard et al., 2015).

\section{Pattern analysis of $f M R I$ data}

Analysis of multivariate patterns evoked during memory retrieval was conducted on functional data from the retrieval runs. In brief, estimates of activation on each trial were computed at every voxel in the brain. These single-trial estimates were then extracted as spatially distributed patterns across relevant ROIs. To compute single-trial estimates of activation, each of the 24 trials in a run was modeled as a separate condition in a GLM. Trials were modeled as 2-TR boxcars beginning at trial onset convolved with a canonical HRF. Mean intensity across the run, linear drift, and estimates of subject motion were modeled as nuisance regressors. The procedure was repeated within each of the 12 runs of the retrieval task, resulting in a GLM estimated for each run and a parameter estimate for each trial in each run in every voxel in the brain.

Patterns of parameter estimates were extracted from all voxels within an ROI, and individual trial patterns were separated according to study condition (overnight/same day/single session) and memory (associative/ item-only). We then measured pattern differentiation as follows: we computed the Pearson correlation between the pattern for each trial and all other trials in the same condition (i.e., a correlation value was computed between each overnight-associative trial and all other overnightassociative trials; LaRocque et al., 2013). We then applied Fisher's $r$-to- $z$ transformation to the between-trial similarities, converted the measures to differentiation by taking $1-z$, and averaged within participant to generate a global measure of differentiation across trials of the same condition. We then used ANOVAs and $t$ tests across participants to measure effects of learning condition and memory performance on neural differentiation.

\section{$\beta$-Series connectivity}

To measure connectivity between hippocampus and MPFC, we used the $\beta$-series method (Rissman et al., 2004; Vilberg and Davachi, 2013). Within each subject, we used the same output of the single-trial GLM model that we used to compute pattern differentiation (see above, Pattern analysis of fMRI data). These single-trial $\beta$ estimates were sorted by condition (overnight and same day) and averaged across voxels within the hippocampal and MPFC ROIs. This yielded a single $\beta$ estimate for each region for each retrieval trial of the experiment. We then computed the Pearson correlation between the $\beta$ estimates across retrieval trials (the $\beta$-series) from hippocampus and MPFC and did so separately within the overnight and same day conditions. To assess the relationship between connectivity and pattern differentiation, we then correlated the difference in $\beta$-series connectivity between the overnight and same day conditions with the difference in pattern similarity for the two conditions.

\section{Results}

\section{Memory performance}

Participants performed well above chance on the Day 7 retrieval test. Collapsing across study condition (overnight/same day/single session) and memory status (associative/item-only), the difference between the hit and false alarm rates for judging words as Old/New was significantly greater than zero $(M=0.60 \pm 0.04$; $\left.t_{(18)}=14.8 ; p<0.001\right)$. Participants also showed high levels of memory for the word-object associations. We compared the proportion of associative trials (collapsing across study list) with an expected mean of 0.5 (i.e., an equal number of associative and item-only trials) and found significantly better associative memory than expected by chance (associative $M=0.74 \pm 0.03$; itemonly $M=0.22 \pm 0.03 ; t_{(18)}=9.0 ; p<0.001$; Fig. $\left.2 A\right)$.

To determine whether associative memory differed by study list, we conducted a one-way ANOVA with study condition (overnight/same day/single session) as a within-participant factor and observed a significant main effect $\left(F_{(2,36)}=55.0 ; p<\right.$ 0.001 ). In addition to our primary conditions of interest (overnight/same day), participants were also trained to criterion on a novel set of word-object associations just before the Day 7 retrieval test (single session condition). Planned comparisons revealed that the proportion of associative memory in the single session condition $(M=0.93 \pm 0.02)$ was significantly greater than in both the overnight $\left(M=0.72 \pm 0.05 ; t_{(18)}=5.35 ; p<\right.$ $0.001)$ and same day $\left(M=0.57 \pm 0.03 ; t_{(18)}=10.9 ; p<0.001\right)$ conditions. This effect was expected given that participants had just learned the single session pairs to criterion before the retrieval test, whereas they had last seen the overnight and same day pairs the week before. Importantly, however, associative memory was also significantly higher in the overnight condition than in the same day condition $\left(t_{(18)}=4.9 ; p<0.001\right)$, demonstrating that distributing learning across $2 \mathrm{~d}$, relative to study within a single day, benefited associative memory retrieval even after 1 week. Thus, we defined the overnight learning benefit as the difference in associative memory between the overnight and same day conditions, and use this measure in later analyses of the fMRI data. Finally, we also examined whether the different study conditions resulted in different levels of forgetting (i.e., Old trials given a New response). A one-way ANOVA with study list as a within-participant factor showed a significant main effect $\left(F_{(2,36)}=\right.$ 
A

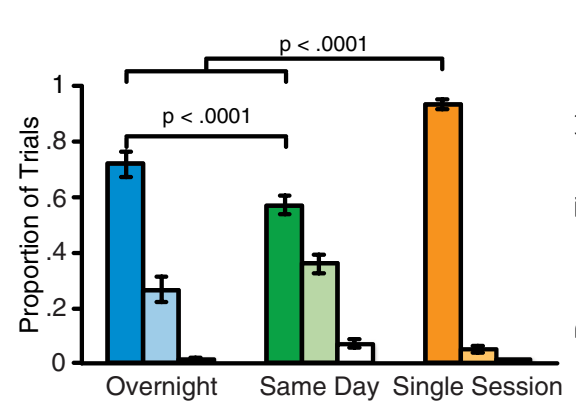

B

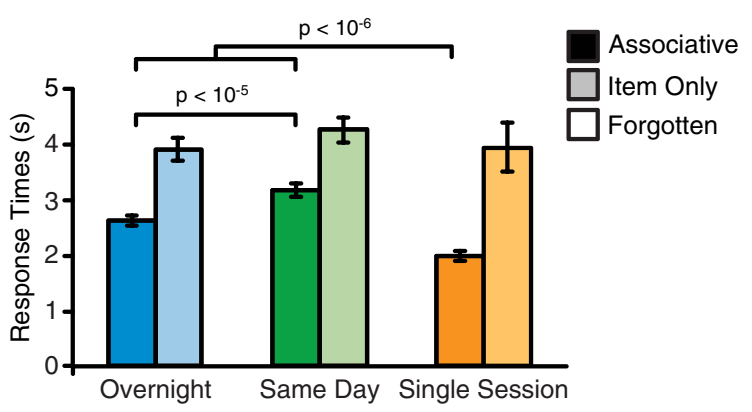

Figure 2. Behavioral data for day 7 retrieval test. $\boldsymbol{A}$, The proportions of associative, item-only and forgotten pairs are plotted for the overnight, same day, and single session lists. Associative memory was higher for overnight pairs compared with same day pairs $\left(p<10^{-4}\right)$, evidence of a distributed learning effect. Associative memory for single session pairs was higher than both overnight and same day pairs (both pairwise comparisons, $p<10^{-4}$ ). $\boldsymbol{B}$, Response times for associative and item-only conditions. In addition to showing higher source memory, participants were also faster to respond for overnight associative memories compared with same day associative memories $\left(p<10^{-5}\right.$ ). Participants were overall fastest for single session associative memories (both pairwise comparisons with overnight and same day trials, $p<10^{-6}$ ). Error bars denote SEM.

$10.9 ; p<0.001)$ that was driven by significantly more forgetting of the same day list $(M=0.07 \pm 0.02)$ compared with the overnight $\left(M=0.02 \pm 0.01 ; t_{(18)}=3.58 ; p=0.002\right)$ and single session $\left(M=0.02 \pm 0.01 ; t_{(18)}=3.33: p=0.004\right)$ lists.

We also analyzed response times to determine whether they varied according to memory and study condition. A comparison of response times for associative and item-only memories, collapsed across study condition, showed that participants were significantly faster to make associative responses than item-only responses (associative $M=2.60 \pm 0.08 \mathrm{~s}$; item-only $M=4.08 \pm$ $0.23 \mathrm{~s} ; t_{(18)}=7.17 ; p<0.001$; Fig. $2 B$ ). Because six participants did not have any single session item-only trials, we focused our analysis on comparing associative response times across study conditions using a one-way ANOVA. The main effect of study condition was significant $\left(F_{(2,36)}=59.1 ; p<0.001\right.$; Fig. $\left.2 B\right)$, and planned comparisons showed that single session associative response times $(M=1.99 \pm 0.08 \mathrm{~s})$ were significantly faster than both overnight $\left(M=2.63 \pm 0.10 \mathrm{~s} ; t_{(18)}=7.09 ; p<0.001\right)$ and same day $\left(M=3.18 \pm 0.12 \mathrm{~s} ; t_{(18)}=8.67, p<0.001\right)$ response times. Finally, overnight response times were also significantly faster than same day response times $\left(t_{(18)}=5.80 ; p<0.001\right)$. Together, object-word pairs learned across $2 \mathrm{~d}$, relative to those learned on a single day, were better and more quickly recalled after 1 week, consistent with the notion that distributing learning across contexts benefited the access to and accuracy of those memory representations.

Finally, although we observed the previously described differences between the overnight and same day conditions on Day 7, these were not attributable to differences between conditions during training. As described in Materials and Methods, both conditions were trained to $90 \%$ criterion. In addition, the conditions did not differ in the number of training rounds required to reach criterion (mean overnight, 3.3; mean same day, 3.2; $p=$ 0.60 , Wilcoxon signed rank test) and in the number of exposures to each item during the training phases on Days 1 and 2 (mean overnight, 7.6; mean same day, 7.5; $p=0.10$, rank sum test). We also found that the difference in the number of presentations did not predict the difference in Day 7 hit rates $\left(r_{(17)}=-0.11 ; p=\right.$ 0.66 , Spearman rank correlation) nor source correct memory $\left(r_{(17)}=-0.07 ; p=0.76\right.$, Spearman rank correlation $)$.

\section{Pattern differentiation in hippocampus}

To measure whether memories are represented in a distinct manner that benefits retrieval, we computed the differentiation in hippocampal multivariate BOLD activity patterns evoked during retrieval of each memory with patterns evoked during retrieval of all the other memories from that learning session. We found that, in left hippocampus, pattern differentiation was significantly higher for associative compared with item-only memories $\left(F_{(1,16)}=11.3 ; p=0.004\right)$ but did not vary by overnight versus same day learning $\left(F_{(1,16)}=2.60 ; p=0.12\right)$, with no interaction between factors $(p=0.58$; Fig. $3 A)$. Planned comparisons confirmed more within-condition hippocampal pattern differentiation for associative trials compared with item-only trials in both the overnight (associative $z=0.90 \pm 0.01$; item-only $z=0.86 \pm$ $0.03 ; t_{(16)}=2.17 ; p=0.04$ ) and same day (associative $z=0.88 \pm$ 0.02 ; item-only $\left.z=0.85 \pm 0.02 ; t_{(16)}=2.36 ; p=0.03\right)$ conditions. We also conducted our pattern differentiation analyses separately for voxels in the anterior and posterior thirds of the left hippocampus, defined by coronal slice position along the anterior/posterior axis. Using a three-way ANOVA (anterior/ posterior $\times$ associative/item-only $\times$ overnight/same day), we found that pattern differentiation in left hippocampus was greater for anterior compared with posterior ROIs (main effect: $F_{(1,16)}=7.05 ; p=0.02$; Fig. $\left.3 B\right)$, greater for associative compared with item-only trials $\left(F_{(1,16)}=7.27 ; p=0.02\right)$, and greater for overnight compared with same day trials $\left(F_{(1,16)}=9.03 ; p=\right.$ $0.008)$. There was a trend for a three-way interaction $(p=0.10)$, but all other interactions with anterior/posterior ROI were not significant $(p>0.41)$.

We also examined right hippocampus, which showed higher differentiation for associative compared with item memories $\left(F_{(1,16)}=14.4 ; p=0.002\right)$ and a trend for greater differentiation with overnight study $\left(F_{(1,16)}=4.09 ; p=0.06\right)$ with a significant difference observed in both the overnight (associative $z=0.89 \pm$ 0.02 ; item-only $\left.z=0.83 \pm 0.03 ; t_{(16)}=2.48 ; p=0.02\right)$ and same day conditions (associative $z=0.87 \pm 0.02$; item-only $z=0.82 \pm$ $\left.0.02 ; t_{(16)}=4.12 ; p<0.001\right)$. There was no interaction between study list and memory $(p=0.76)$. These results provide evidence for hippocampal pattern separation during a 1 week memory test that is related to successful associative memory retrieval. When comparing anterior/posterior ROIs in right hippocampus, we also found a main effect such that pattern differentiation was greater in anterior compared with posterior right hippocampus $\left(F_{(1,16)}=9.81 ; p=0.006\right.$; Fig. $\left.3 B\right)$ and greater for associative compared with item-only trials $\left(F_{(1,16)}=13.0 ; p=0.002\right)$. There were no other main effects or interactions (all $p$ values $>0.12$ ). 
A

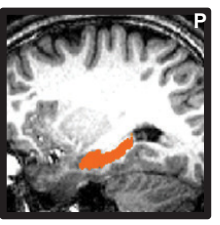

Left Hippocampus
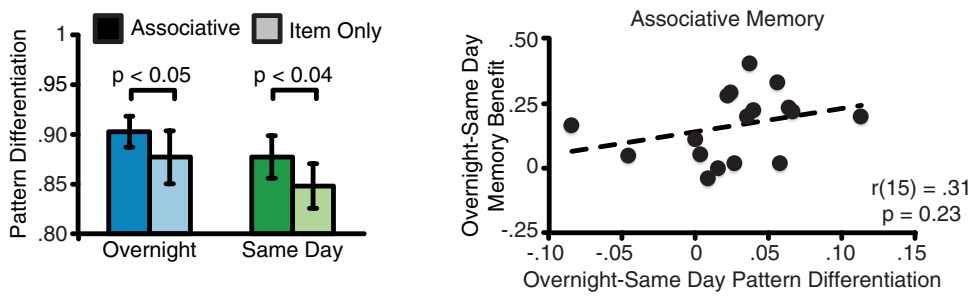

B
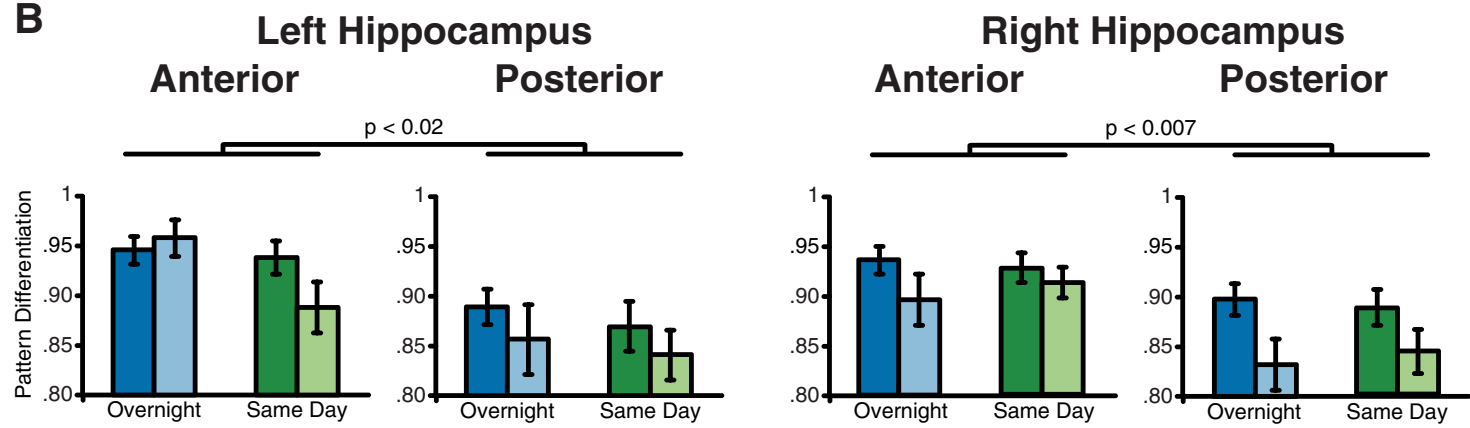

Figure 3. Hippocampal pattern differentiation. $\boldsymbol{A}$, Left, Example subject left hippocampus anatomical region of interest. Middle, In left hippocampus, differentiation did not vary between overnight and same day memory ( $p=0.12$ ) but was significantly higher for associative compared with item-only memory (main effect, $p=0.004$ ), a difference that was significant within the overnight and same day conditions individually. Right, The difference in overnight/same day pattern differentiation in hippocampus did not correlate with the overnight memory benefit ( $p=0.23$ ). $\boldsymbol{B}$, Left, In left hippocampus pattern differentiation was significantly greater in anterior compared with posterior regions $(p=0.02)$. Right, In right hippocampus, pattern differentiation was also significantly greater for anterior compared with posterior regions $(p=0.006)$.

To determine whether the level of pattern differentiation in hippocampal memory representations was related to memory performance, we correlated overnight $>$ same day associative memory differentiation with the overnight learning benefit. We also conducted the same analysis between overnight $>$ same day item-only memory differentiation and the overnight $>$ same day item-only behavioral memory difference. In both left and right hippocampus, neither the associative correlation (left: $r_{(15)}=$ $0.31, p=0.23$; Fig. $3 A$; right: $\left.r_{(15)}=0.30, p=0.24\right)$ nor the item-only correlation (left: $r_{(15)}=-0.28, p=0.27$; right: $r_{(15)}=$ $0.32, p=0.21)$ was significant.

In summary, pattern separation in hippocampus was higher for retrieved memories that contained associated source information compared with memories containing only item information, regardless of whether information was learned the week before across $24 \mathrm{~h}$ or within the same session. We observed greater differentiation in anterior compared with posterior hippocampus, which highlights a dissociation along the long axis of the hippocampus (also see Tompary and Davachi, 2017) but is not fully consistent with models that predict more separated representations in posterior compared with anterior subregions (Poppenk et al., 2013). In contrast, we do think the current findings are consistent with a role for anterior hippocampus in a distributed network that represents specific item details in episodic memory (Ranganath and Ritchey, 2012).

\section{Pattern differentiation in MPFC}

As in left hippocampus, there was significantly more differentiation in MPFC for associative memories compared with item-only memories $\left(F_{(1,16)}=9.45, p=0.007\right)$. Interestingly, however, unlike the results from hippocampus, there was a significant interaction between study list (overnight/same day) and memory $\left(F_{(1,16)}=7.39, p=0.02\right)$ that was driven by a difference in associative/item-only memory specific to the overnight list (as- sociative $z=0.92 \pm 0.02$; item-only $z=0.84 \pm 0.03 ; t_{(16)}=3.18$; $p=0.006$ ), with no difference in associative/item-only memory for the same day list ( $p=0.16$; Fig. $4 A$ ). A direct comparison of associative trials in the overnight and same day conditions showed that overnight differentiation was higher than same day differentiation (same day $z=0.88 \pm 0.02 ; t_{(16)}=3.70$; $p=0.002$ ).

We next investigated whether MPFC differentiation was related to the behavioral enhancement seen for overnight associative memory relative to same day associative memory. We found that overnight $>$ same day pattern differentiation in MPFC for associative memory trials was significantly correlated with the overnight learning benefit $\left(r_{(15)}=0.53 ; p=0.03\right.$; Fig. $\left.4 A\right)$. Thus, greater differentiation in evoked MPFC neural patterns for the overnight condition relative to the same day condition was associated with increases in overnight associative memory, relative to same day associative memory (this correlation did not differ from the hippocampal correlation, $p=0.18$ ). There was no correlation between overnight $>$ same day differentiation for item-only memories and overnight $>$ same day item-only memory success $\left(r_{(15)}=0.38 ; p=0.13\right)$, but this did not differ from the associative correlation $(p=0.30)$. These data show that, relative to same day memories, greater pattern differentiation in MPFC patterns across overnight associative memories predicts a larger overnight learning benefit. More broadly, these data are consistent with the idea that differentiation in measured BOLD retrieval patterns in MPFC supports enhancements in associative memory derived from studying information across an overnight delay.

One explanation for the differentiation of retrieval patterns in MPFC is simply that overnight memories are tagged during encoding with information from two contexts, which is then reinstated at retrieval. If this were the case, then one prediction that would follow is that overnight memories should be more likely to cluster into two groups than same day memories. To test this, we 

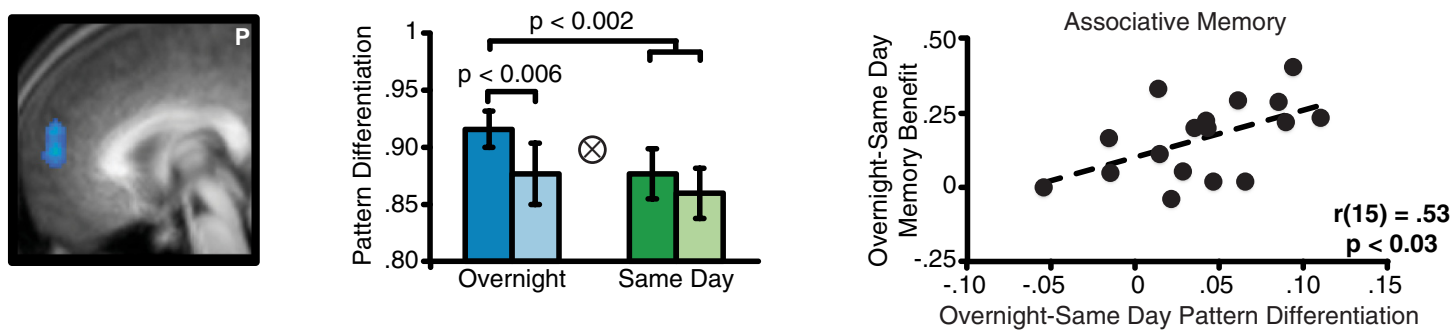

B

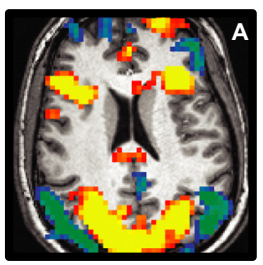

Task Active Voxels

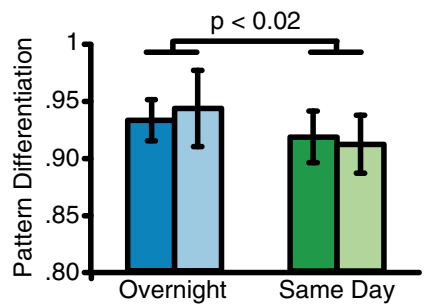

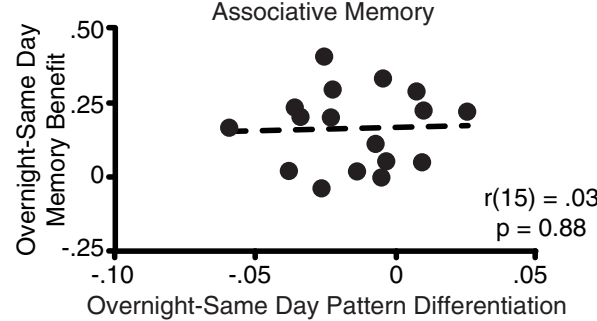

Figure 4. MPFC and brain-wide pattern differentiation. $A$, Left, MPFC region of interest rendered on the group-average brain. Middle, In MPFC, there was significantly more differentiation for associative versus item-only memories only within the overnight condition $(p=0.006)$ but not in the same day condition $(p>0.16$; interaction $p=0.02)$. Differentiation for overnight associative trials was also significantly greater than for same day associative memories $(p=0.002)$. Error bars denote SEM. The $\otimes$ symbol indicates significant interaction $(p<0.05)$. Right, The difference in overnight/same day pattern differentiation on Day 7 correlated with the overnight memory benefit $\left(r_{(15)}=0.53 ; p=0.03\right)$. $\boldsymbol{B}$, Left, Example subject brain-wide task active mask. Middle, Pattern differentiation was greater for overnight than same day retrieval $(p<0.02)$ but did not differ as a function of memory success. Error bars denote SEM. Right, The difference in overnight/same day pattern differentiation on Day 7 was not correlated with the overnight memory benefit $\left(r_{(15)}=0.03 ; p=0.88\right)$.

applied $k$-means clustering to segment the overnight and same day patterns for each subject into two clusters. We then assessed whether the between-cluster distance for the overnight patterns was larger than for the same day patterns, as would be the case if there was greater evidence for two distinct clusters in the overnight condition. In MPFC, we did not find greater evidence for two clusters in the overnight compared with same day conditions (mean within vs between-cluster correlation: overnight, 0.22; same day, $\left.0.21 ; t_{(16)}=1.21 ; p=0.24\right)$; there was also no difference in the left hippocampus (overnight, 0.17; same day, 0.19; $\left.t_{(16)}=-1.02 ; p=0.32\right)$.

To confirm that this null finding was not the result of lack of power to discriminate conditions of interest, we repeated the analysis and used it to cluster associative and item-only patterns into groups, as a way to classify the two trial types. We assessed classification for each subject using area under the curve (AUC) and compared the AUC distribution across subjects with chance (0.50). We found that the mean of the group AUC distribution was significantly above chance $(M=0.55 ; p<0.001)$, confirming that the analysis was sufficiently powered to separate associative and item-only MPFC patterns. Together, these results suggest that greater MPFC differentiation was not necessarily driven by the direct representation variable encoding of contextual information during learning.

\section{Brain-wide pattern differentiation}

We were also interested in whether large-scale activity distributed across the brain exhibited similar patterns as observed in hippocampus and MPFC. For each subject, we defined a mask of the 5000 most active voxels in a contrast of Task $>$ Baseline and computed pattern differentiation across this distributed set of voxels (single-subject example; Fig. 4B). Unlike the hippocampus and MPFC, brain-wide pattern differentiation did not differ based on memory retrieval success (associative vs item-only main effect: $\left.F_{(1,16)}=0.11 ; p=0.74\right)$ but was higher overall for pairs learned overnight compared with same day $\left(F_{(1,16)}=7.89 ; p=\right.$ 0.01; Fig. 4B).

When comparing pattern differentiation across the three ROIs (MPFC/hippocampus/ brain-wide), we found that the interaction of region $\times$ study list (overnight/same day) $\times$ memory $\left(F_{(2,32)}=2.82 ; p=0.07\right)$ was driven by greater study list and memory-dependent pattern differentiation in MPFC than over the distributed task active brain mask $(p=0.03)$. In left hippocampus, study list and memory-dependent pattern differentiation did not differ from that observed in the task active brain mask $(p=0.29)$.

As in MPFC and hippocampus, we next examined whether the difference in pattern differentiation for overnight compared with same day pairs predicted the overnight/same day behavioral enhancement in associative memory performance. Brain-wide pattern differentiation was not significantly correlated with the behavioral enhancement $\left(r_{(15)}=0.03 ; p=0.88\right.$; Fig. $\left.4 B\right)$ and showed a trend for being lower than observed in MPFC (permutation test, $p=0.08$ ).

MPFC-hippocampal connectivity predicts differentiation To shed light on the mechanism by which MPFC representations of overnight memories were more differentiated than same day memories, we measured $\beta$-series connectivity between hippocampus and MPFC during correct associative retrieval. We 




Figure 5. Hippocampal-MPFC connectivity predicts MPFC pattern differentiation. The difference in hippocampal-MPFC connectivity between the overnight/same day conditions correlates with the level of pattern differentiation in $\operatorname{MPFC}\left(r_{(15)}=0.60 ; p<0.02\right)$.

hypothesized that if context-based MPFC differentiation arises from reorganization in mnemonic networks (Siapas and Wilson, 1998; Takashima et al., 2009; van Kesteren et al., 2010), then connectivity with hippocampus may be related to this mnemonic differentiation in MPFC. Indeed, we found that, across participants, the difference in hippocampal-MPFC connectivity for overnight memories relative to same day memories correlated with the increase in representational differentiation for overnight compared with same day memories in MPFC $\left(r_{(15)}=0.60 ; p=\right.$ 0.01 ; Fig. 5). Thus, those participants who exhibited greater hippocampal-MPFC connectivity during retrieval of overnight memories compared with same day memories also showed more pattern separation for overnight compared with same day memories in MPFC. This suggests that study across an overnight delay leads to enhanced hippocampal-MPFC interactions that may support the development of differentiated memory representations over time.

\section{Univariate effects in left hippocampus and MPFC}

Although we were focused on assessing pattern differentiation, we also examined univariate activity in our primary regions of interest. Specifically, we tested whether parameter estimates in left hippocampus and MPFC significantly differed for the overnight/same day and associative/item-only conditions. A $2 \times 2$ ANOVA applied to the data from the left hippocampus (Fig. 6A) showed no significant main effects or interaction (all $p$ values $>0.12$ ). Figure $6 B$ shows that MPFC was more active for the overnight compared with the same day condition $\left(F_{(1,16)}=8.27\right.$; $p=0.01)$ and also shows a study condition $\times$ memory interaction $\left(F_{(1,16)}=6.73 ; p=0.02\right)$. A $3 \times 2$ ANOVA comparing the two ROIs showed a significant main effect of region $\left(F_{(1,16)}=\right.$ 138 ; $p<0.001$ ) but no interactions of region with study condition or memory.

\section{Discussion}

Understanding the mechanisms that promote the longevity of learning is a critical focus of cognitive neuroscience. We find that learning word-object pairs across the distinct temporal contexts of $2 \mathrm{~d}$ benefited long-term associative memory retrieval compared with word-object pairs learned on $1 \mathrm{~d}$, even though both overnight and same day associations were trained to the same high learning criterion (90\%) and were matched in the number of training sessions. At 1 week retrieval, multivariate activity patterns in MPFC were more differentiated for associations learned across temporal contexts compared with associations learned within a single temporal context. Furthermore, the increase in pattern separation for overnight compared with same day memories in MPFC correlated with the behavioral memory benefit for the overnight memories, suggesting that differentiation of MPFC retrieval patterns contributes to the memory benefit for information studied across contexts. We also found a correlation between context-driven pattern differentiation of MPFC activity for individual memories and functional connectivity between hippocampus and MPFC, suggesting that persistent hippocampalcortical connectivity 1 week after learning supports the observed MPFC differentiation. These data show that robust long-term memory is supported by differentiation in the neural activity patterns that represent memories and suggest that this differentiation arises as a consequence of context change during learning that promotes sustained MPFC-hippocampal interactions.

Given that participants encoded overnight pairs on $2 \mathrm{~d}$ and same day pairs on a single day, one explanation for greater MPFC pattern differentiation for the overnight condition is that participants encoded, and subsequently retrieved, associative details from two temporal contexts (Estes, 1955). One prediction that might follow from such a model is that overnight patterns should be more likely to cluster into two groups than same day memories, perhaps reflecting differential levels of contextual associations across memories. However, we did not find evidence that overnight memories were more likely to cluster into two groups than same day memories, suggesting that MPFC pattern differentiation may arise as a consequence of repeated learning across days but does not directly reflect variability in contextual associations across conditions. Repeated learning across contexts may facilitate the long-term retention of unique memory traces through mechanisms that enhance the fidelity of individual memory representations (Thios and D'Agostino, 1976; Karlsson Wirebring et al., 2015).

Future work could investigate the degree to which the night of sleep itself (in the overnight condition) is important for promoting differentiation. We observed differentiation for overnight compared with same day memories despite the fact that both types of memories were matched in several ways. First, both types were trained to the same high behavioral criterion. Second, both overnight and same day memories had the opportunity for significant consolidation to occur during the 1 week between study and retrieval. These unique features of our design highlight the important role that reactivation after a $24 \mathrm{~h}$ period played in the overnight condition. One possibility is that the overnightdelayed reactivation tagged overnight memories with enhanced behavioral relevance, leading these memories to undergo enhanced consolidation the following week (Singer and Frank, 2009; Lesburguères et al., 2011; Wilhelm et al., 2011; Oudiette et al., 2013). Another possibility is that one night of sleep before restudy establishes a schema (in the sense of a pre-existing neural network), which then promotes additional learning after the night of sleep (Tse et al., 2007, 2011; van Kesteren et al., 2012). Each of these studies includes some period of rest before reactivation, which appears to be critical in enhancing memory retention (Litman and Davachi, 2008). Thus, future work that can compare representations at encoding, rest, and retrieval over extended time scales will be needed to adjudicate between these and other possibilities.

The observation that distributing study episodes across time is beneficial for memory relative to massing study within a shorter time span is one of the most robust findings in the study of memory (Ruch, 1928; McGeoch and Irion, 1952; Ebbinghaus, 


\section{A}

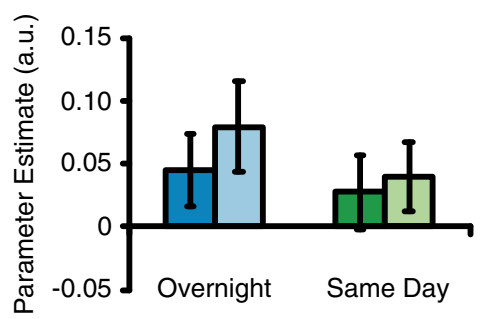

B

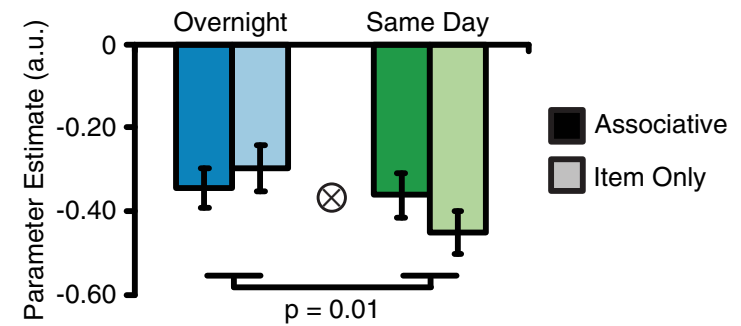

Figure 6. Univariate activity in hippocampus and MPFC. $\boldsymbol{A}$, We did not observe significant modulation of univariate activity in left hippocampus across conditions (all $p$ values $>0.12$ ). $\boldsymbol{B}$, In MPFC, there was a main effect of overnight/same day condition $(p=0.01)$ as well as an interaction $(p=0.02)$. Error bars denote SEM. The $\otimes$ symbol indicates significant interaction $(p<0.05)$.

1964; Dempster, 1989; Cepeda et al., 2006; Litman and Davachi, 2008). Our behavioral results are consistent with this literature, showing that object-word associations were better remembered 1 week later if initially learned in two sessions distributed across $2 \mathrm{~d}$ than associations learned in two sessions within the same day. Our design equated the number of learning sessions across the overnight and same day lists, as well as the level of immediate memory for the two lists. Thus, our data suggest that the memory benefit for the overnight condition 1 week later is attributable to learning across $2 \mathrm{~d}$. Furthermore, the fMRI results highlight a potential mechanism for the efficacy of distributed learning, namely that it promotes efficient, differentiated neural representations of memories that facilitates successful retrieval.

Prior fMRI work has suggested that the extent to which patterns overlap (pattern similarity) across repeated learning trials of the same stimulus is related to successful recognition memory. This work has shown that repeated individual stimuli that are later remembered were associated with greater similarity in the evoked fMRI activity patterns across encoding repetitions (Xue et al., 2010, 2013; Watanabe et al., 2011; Gordon et al., 2013; Ward et al., 2013). Similarity across trial-unique, same-category stimuli during encoding is predictive of memory success (Kuhl et al., 2012), as is similarity between patterns present during encoding and retrieval (Zeithamova and Preston, 2010; Ritchey et al., 2013; Danker et al., 2016; Tompary et al., 2016), again suggesting the important role that similarity plays in processing the same events in memory. This general approach is distinct from the one we adopt here in which we measure how memories are represented compared with one another: an inter-item similarity analysis. Prior work using a similar approach suggests that both similarity and differentiation during encoding support later memory but are instantiated in distinct brain areas, the MTL cortex and hippocampus, that support slow extraction of commonalities across memories versus rapid differentiation of overlapping experiences (Norman and O'Reilly, 2003; LaRocque et al., 2013; Tompary and Davachi, 2017). It is possible that enhanced similarity across learning repetitions of the same item could promote differentiation across items at retrieval (i.e., our primary neural measure) by sharpening the representations of individual memories relative to one another, following repeated study/test attempts during learning. Testing-related neural differentiation is known to predict memory performance after a 1 week delay (Karlsson Wirebring et al., 2015). Therefore, one critical question for future research is to examine the extent to which repeated study supports later memory through engagement of similarity-based mechanisms, whereas repeated testing does so through differentiation-based mechanisms.

The present work extends our knowledge of how MPFC represents contextual information over time. Previous work showed that differentiation in MPFC BOLD activity patterns at encoding predicts temporal order memory (Jenkins and Ranganath, 2016). MPFC activity is also enhanced during retrieval of temporally remote memories compared with recent memories (Takashima et al., 2006; Gais et al., 2007; Sterpenich et al., 2009; Nieuwenhuis and Takashima, 2011). Furthermore, multivariate classification of MPFC BOLD patterns is better for remote than recent autobiographical memories, suggesting remote memory representations are more differentiated (Bonnici et al., 2012). Our work connects these data on MPFC encoding and retrieval activity by showing that MPFC pattern differentiation is related to the fidelity of individual associative memories after 1 week, when those associations were learned across temporal contexts. Furthermore, our univariate analysis showing greater MPFC activity in the overnight compared with same day condition is consistent with a role for MPFC in retrieval of consolidated memories and extends prior work by contrasting conditions that are matched in terms of remoteness.

Given that we scanned only at retrieval, an open question concerns the time course by which differentiation across memory representations emerges after study (Bonnici and Maguire, 2018). Understanding the time line will help distinguish between the influence of processes engaged during encoding, retrieval, or in between (Hulbert and Norman, 2015). For example, future work in which the encoding phase is scanned could determine whether differences in neural representation emerge during study. This work will be important to determine the relationship between the context-based differentiation we observed and other mechanisms such as study-phase retrieval (Thios and D'Agostino, 1976; Braun and Rubin, 1998). Neural markers of memory stabilization in cortical networks have also been identified shortly after encoding of a to-be-remembered experience (Tambini et al., 2010; Tambini and Davachi, 2013; Ben-Yakov and Dudai, 2011; Tompary et al., 2015). Hippocampal-cortical connectivity after a $1 \mathrm{~d}$ delay predicts subsequent resistance to forgetting (Vilberg and Davachi, 2013), and persistent connectivity between hippocampus and MPFC during the 1 week delay is a potential mechanism that could lead to later pattern differentiation as representations become established in cortical networks (Peyrache et al., 2009; Richards et al., 2014). Our data showing hippocampal-MPFC connectivity predicting neural differentiation in MPFC are consistent with this hypothesis, but future work will be needed to address how such connectivity emerges and evolves over time.

Prominent theories propose that MPFC contributes to longterm memory by supporting the integration of new information into established memory networks (Tse et al., 2007; van Kesteren et al., 2012), reducing forgetting of new information by providing 
a set of stable associations that can be used to access specific memories during later retrieval. A computational challenge that arises when integrating new information into established networks is avoiding interference between similar representations (McClelland et al., 1995; O'Reilly and Rudy, 2001). Our findings suggest that one way to avoid such interference is to use contextual information to differentiate similar input representations, a notion that has figured prominently in theoretical and experimental work on the role of hippocampus in episodic memory encoding (Marr, 1971; Norman and O'Reilly, 2003; Leutgeb et al., 2004, 2007; Bakker et al., 2008; LaRocque et al., 2013; Hulbert and Norman, 2015). Our data show that distributing study context across $2 \mathrm{~d}$ leads to differentiation in activity patterns in MPFC. The extent of this differentiation predicts the increase in memory performance that results from distributed study and is related to functional connectivity between MPFC and hippocampus. These data implicate a role for MPFC in representing contextual information in long-term memory over long time scales and provide novel evidence that distributed study leads to greater memory retention via persistent hippocampal-MPFC interactions.

\section{References}

Bakker A, Kirwan CB, Miller M, Stark CE (2008) Pattern separation in the human hippocampal CA3 and dentate gyrus. Science 319:1640-1642. CrossRef Medline

Ben-Yakov A, Dudai Y (2011) Constructing realistic engrams: poststimulus activity of hippocampus and dorsal striatum predicts subsequent episodic memory. J Neurosci 31:9032-9042. CrossRef Medline

Bonnici HM, Maguire EA (2018) Two years later-revisiting autobiographical memory representations in vmPFC and hippocampus. Neuropsychologia 110:159-169. CrossRef Medline

Bonnici HM, Chadwick MJ, Lutti A, Hassabis D, Weiskopf N, Maguire EA (2012) Detecting representations of recent and remote autobiographical memories in vMPFC and hippocampus. J Neurosci 32:16982-16991. CrossRef Medline

Braun K, Rubin DC (1998) The spacing effect depends on an encoding deficit, retrieval, and time in working memory: evidence from oncepresented words. Memory 6:37-65. CrossRef Medline

Cepeda N, Pashler H, Vul E, Wixted J, Rohrer D (2006) Distributed practice in verbal recall tasks: a review and quantitative synthesis. Psychol Bull 132:354-380. CrossRef

Danker JF, Tompary A, Davachi L (2016) Trial-by-trial hippocampal encoding activation predicts the fidelity of cortical reinstatement during subsequent retrieval. Cereb Cortex 27:3515-3524. CrossRef Medline

Dempster F (1989) Spacing effects and their implications for theory and practice. Educ Psychol Rev 1:309-330. CrossRef

DuBrow S, Davachi L (2014) Temporal memory is shaped by encoding stability and intervening item reactivation. J Neurosci 34:13998-14005. CrossRef Medline

Ebbinghaus H (1964) Memory: a contribution to experimental psychology (Ruger HA, Bussenius CE, eds). New York: Dover.

Estes WK (1955) Statistical theory of spontaneous recovery and regression. Psychol Rev 62:145-154. CrossRef Medline

Ezzyat Y, Davachi L (2014) Similarity breeds proximity: pattern similarity within and across contexts is related to later mnemonic judgments of temporal proximity. Neuron 81:1179-1189. CrossRef Medline

Gais S, Albouy G, Boly M, Dang-Vu TT, Darsaud A, Desseilles M, Rauchs G, Schabus M, Sterpenich V, Vandewalle G, Maquet P, Peigneux P (2007) Sleep transforms the cerebral trace of declarative memories. Proc Natl Acad Sci U S A 104:18778-18783. CrossRef Medline

Gordon AM, Rissman J, Kiani R, Wagner AD (2013) Cortical reinstatement mediates the relationship between content-specific encoding activity and subsequent recollection decisions. Cereb Cortex 24:3350-3364. Medline

Guise KG, Shapiro ML (2017) Medial prefrontal cortex reduces memory interference by modifying hippocampal encoding. Neuron 94:183192.e8. CrossRef Medline

Heusser AC, Poeppel D, Ezzyat Y, Davachi L (2016) Episodic sequence memory is supported by a theta-gamma phase code. Nat Neurosci 19: 1374-1380. CrossRef Medline
Howard MW, Kahana MJ (2002) A distributed representation of temporal context. J Math Psychol 46:269-299. CrossRef

Howard MW, Shankar KH, Aue WR, Criss AH (2015) A distributed representation of internal time. Psychol Rev 122:24-53. CrossRef Medline

Hsieh LT, Gruber MJ, Jenkins LJ, Ranganath C (2014) Hippocampal activity patterns carry information about objects in temporal context. Neuron 81:1165-1178. CrossRef Medline

Hulbert JC, Norman KA (2015) Neural differentiation tracks improved recall of competing memories following interleaved study and retrieval practice. Cereb Cortex 25:3994-4008. CrossRef Medline

Hyman JM, Ma L, Balaguer-Ballester E, Durstewitz D, Seamans JK (2012) Contextual encoding by ensembles of medial prefrontal cortex neurons. Proc Natl Acad Sci U S A 109:5086-5091. CrossRef Medline

Insausti R, Juottonen $\mathrm{K}$, Soininen $\mathrm{H}$, Insausti AM, Partanen $\mathrm{K}$, Vainio $\mathrm{P}$, Laakso MP, Pitkanen A (1998) MR volumetric analysis of the human entorhinal, perirhinal, and temporopolar cortices. Am J Neuroradiol 19: 659-671. Medline

Jenkins LJ, Ranganath C (2016) Distinct neural mechanisms for remembering when an event occurred. Hippocampus 26:554-559. CrossRef Medline

Karlsson Wirebring L, Wiklund-HörnqvistC, Eriksson J, Andersson M, Jonsson B, Nyberg L (2015) Lesser neural pattern similarity across repeated tests is associated with better long-term memory retention. J Neurosci 35:9595-9602. CrossRef Medline

Kitamura T, Ogawa SK, Roy DS, Okuyama T, Morrissey MD, Smith LM, Redondo RL, Tonegawa S (2017) Engrams and circuits crucial for systems consolidation of a memory. Science 356:73-78. CrossRef Medline

Kuhl BA, Rissman J, Wagner AD (2012) Multi-voxel patterns of visual category representation during episodic encoding are predictive of subsequent memory. Neuropsychologia 50:458-469. CrossRef Medline

LaRocque KF, Smith ME, Carr VA, Witthoft N, Grill-Spector K, Wagner AD (2013) Global similarity and pattern separation in the human medial temporal lobe predict subsequent memory. J Neurosci 33:5466-5474. CrossRef Medline

Lesburguères E, Gobbo OL, Alaux-Cantin S, Hambucken A, Trifilieff $\mathrm{P}$, Bontempi B (2011) Early tagging of cortical networks is required for the formation of enduring associative memory. Science 331:924-928. CrossRef Medline

Leutgeb JK, Leutgeb S, Moser, MB, Moser EI (2007) Pattern separation in the dentate gyrus and CA3 of the hippocampus. Science 315:961-966. CrossRef Medline

Leutgeb S, Leutgeb JK, Treves A, Moser MB, Moser EI (2004) Distinct ensemble codes in hippocampal areas CA3 and CA1. Science 305:12951298. CrossRef Medline

Litman L, Davachi L (2008) Distributed learning enhanced relational memory consolidation. Learn Mem 15:711-716. CrossRef Medline

Long NM, Sperling MR, Worrell GA, Davis KA, Gross RE, Lega BC, Jobst BC, Sheth SA, Zaghloul K, Stein JM, Kahana MJ (2017) Contextually mediated spontaneous retrieval is specific to the hippocampus. Curr Biol 27: 1074-1079. CrossRef Medline

Mankin EA, Sparks FT, Slayyeh B, Sutherland RJ, Leutgeb S, Leutgeb JK (2012) Neuronal code for extended time in the hippocampus. Proc Natl Acad Sci U S A 109:19462-19467. CrossRef Medline

Manns JR, Howard MW, Eichenbaum H (2007) Gradual changes in hippocampal activity support remembering the order of events. Neuron 56 : 530-540. CrossRef Medline

Marr D (1971) Simple memory: a theory for archicortex. Philos Trans R Soc Lond B Biol Sci 262:23-81. CrossRef Medline

McClelland JL, McNaughton BL, O’Reilly RC (1995) Why there are complementary learning systems in the hippocampus and neocortex: insights from the successes and failures of connessionist models of learning and memory. Psychol Rev 102:419-457. CrossRef Medline

McGeoch J, Irion A (1952) The distribution of practice and reminiscence. In: The psychology of human learning, Ed 2, pp 138-193. New York: David McKay.

Nieuwenhuis IL, Takashima A (2011) The role of the ventromedial prefrontal cortex in memory consolidation. Behav Brain Res 218:325-334. CrossRef Medline

Norman KA, O’Reilly RC (2003) Modeling hippocampal and neocortical contributions to recognition memory: a complementary-learning-systems approach. Psychol Rev 110:611-646. CrossRef Medline

O'Reilly RC, Rudy JW (2001) Conjunctive representations in learning and 
memory: principles of cortical and hippocampal function. Psychol Rev 108:311-345. CrossRef Medline

Oudiette D, Antony JW, Creery JD, Paller KA (2013) The role of memory reactivation during wakefulness and sleep in determining which memories endure. J Neurosci 33:6672-6678. CrossRef Medline

Peyrache A, Khamassi M, Benchenane K, Wiener SI, Battaglia FP (2009) Replay of rule-learning related neural patterns in the prefrontal cortex during sleep. Nat Neurosci 12:919-926. CrossRef Medline

Polyn SM, Norman KA, Kahana MJ (2009a) A context maintenance and retrieval model of organizational processes in free recall. Psychol Rev 116:129-156. CrossRef Medline

Polyn SM, Norman KA, Kahana MJ (2009b) Task context and organization in free recall. Neuropsychologia 47:2158-2163. CrossRef Medline

Poppenk J, Evensmoen HR, Moscovitch M, Nadel L (2013) Long-axis specialization of the human hippocampus. Trends Cogn Sci 17:230-240. CrossRef Medline

Ranganath C, Ritchey M (2012) Two cortical systems for memory-guided behaviour. Nat Rev Neurosci 13:713-726. CrossRef Medline

Richards BA, Xia F, Santoro A, Husse J, Woodin MA, Josselyn SA, Frankland PW (2014) Patterns across multiple memories are identified over time. Nat Neurosci 17:981-986. CrossRef Medline

Rissman J, Gazzaley A, D’Esposito M (2004) Measuring functional connectivity during distinct stages of a cognitive task. Neuroimage 23:752-763. CrossRef Medline

Ritchey M, Wing EA, LaBar KS, Cabeza R (2013) Neural similarity between encoding and retrieval is related to memory via hippocampal interactions. Cereb Cortex 23:2818-2828. Medline

Ritchey M, Montchal ME, Yonelinas AP, Ranganath C (2015) Delaydependent contributions of medial temporal lobe regions to episodic memory retrieval. Elife 4:e05025. CrossRef

Ruch T (1928) Factors influencing the relative economy of massed and distributed practice in learning. Psychol Rev 35:19-45. CrossRef

Schlichting ML, Preston AR (2016) Hippocampal-medial prefrontal circuit supports memory updating during learning and post-encoding rest. Neurobiol Learn Mem 134:91-106. CrossRef Medline

Siapas AG, Wilson MA (1998) Coordinated interactions between hippocampal ripples and cortical spindles during slow-wave sleep. Neuron 21:1123-1128. CrossRef Medline

Siapas AG, Lubenov EV, Wilson MA (2005) Prefrontal phase locking to hippocampal theta oscillations. Neuron 46:141-151. CrossRef Medline

Singer AC, Frank LM (2009) Rewarded outcomes enhance reactivation of experience in the hippocampus. Neuron 64:910-921. CrossRef Medline

Sterpenich V, Albouy G, Darsaud A, Schmidt C, Vandewalle G, Dang Vu TT, Desseilles M, Phillips C, Degueldre C, Balteau E, Collette F, Luxen A, Maquet P (2009) Sleep promotes the neural reorganization of remote emotional memory. J Neurosci 29:5143-5152. CrossRef Medline

Takashima A, Nieuwenhuis IL, Rijpkema M, Petersson KM, Jensen O, Fernández G (2007) Memory trace stabilization leads to large-scale changes in the retrieval network: a functional MRI study on associative memory. Learning \& Memory 14:472-479. CrossRef Medline

Takashima A, Petersson KM, Rutters F, Tendolkar I, Jensen O, Zwarts MJ, McNaughton BL, Fernández G (2006) Declarative memory consolidation in humans: a prospective functional magnetic resonance imaging study. Proc Natl Acad Sci U S A 103:756-761. CrossRef Medline

Takashima A, Nieuwenhuis IL, Jensen O, Talamini LM, Rijpkema M, Fernández G (2009) Shift from hippocampal to neocortical centered retrieval network with consolidation. J Neurosci 29:10087-10093. CrossRef Medline

Tambini A, Davachi L (2013) Persistence of hippocampal multivoxel patterns into postencoding rest is related to memory. Proceedings of the National Academy of Sciences 110:19591-19596. CrossRef

Tambini A, Ketz N, Davachi L (2010) Enhanced brain correlations during rest are related to memory for recent experiences. Neuron 65:280-290. CrossRef Medline

Thios SJ, D'Agostino PR (1976) Effects of repetition as a function of studyphase retrieval. J Verbal Learn Verbal Behav 15:529-536. CrossRef

Tompary A, Davachi L (2017) Consolidation promotes the emergence of representational overlap in the hippocampus and medial prefrontal cortex. Neuron 96:228-241.e5. CrossRef Medline

Tompary A, Duncan K, Davachi L (2015) Consolidation of associative and item memory is related to post-encoding functional connectivity between the ventral tegmental area and different medial temporal lobe subregions during an unrelated task. J Neurosci 35:7326-7331. CrossRef Medline

Tompary A, Duncan K, Davachi L (2016) High-resolution investigation of memory-specific reinstatement in the hippocampus and perirhinal cortex. Hippocampus 26:995-1007. CrossRef Medline

Tse D, Langston RF, Kakeyama M, Bethus I, Spooner PA, Wood ER, Witter MP, Morris R (2007) Schemas and memory consolidation. Science 316: 76-82. CrossRef Medline

Tse D, Takeuchi T, Kakeyama M, Kajii Y, Okuno H, Tohyama C, Morris RGM (2011) Schema-dependent gene activation and memory encoding in neocortex. Science 333:891-895. CrossRef Medline

van Kesteren MT, Fernández G, Norris DG, Hermans EJ (2010) Persistent schema-dependent hippocampal-neocortical connectivity during memory encoding and postencoding rest in humans. Proc Natl Acad Sci U S A 107:7550-7555. CrossRef Medline

van Kesteren MTR, Ruiter DJ, Fernández G, Henson RN (2012) How schema and novelty augment memory formation. Trends Neurosci 35: 211-219. CrossRef

Vilberg KL, Davachi L (2013) Perirhinal-hippocampal connectivity during reactivation is a marker for object-based memory consolidation. Neuron 79:1232-1242. CrossRef Medline

Ward EJ, Chun MM, Kuhl BA (2013) Repetition suppression and multivoxel pattern similarity differentially track implicit and explicit visual memory. J Neurosci 33:14749-14757. CrossRef Medline

Watanabe T, Hirose S, Wada H, Katsura M, Chikazoe J, Jimura K, Imai Y, Machida T, Shirouzu I, Miyashita Y, Konishi S (2011) Prediction of subsequent recognition performance using brain activity in the medial temporal lobe. Neuroimage 54:3085-3092. CrossRef Medline

Wilhelm I, Diekelmann S, Molzow I, Ayoub A, Mölle M, Born J (2011) Sleep selectively enhances memory expected to be of future relevance. J Neurosci 31:1563-1569. CrossRef Medline

Xue G, Dong Q, Chen C, Lu Z, Mumford JA, Poldrack RA (2010) Greater neural pattern similarity across repetitions is associated with better memory. Science 330:97-101. CrossRef Medline

Xue G, Dong Q, Chen C, Lu ZL, Mumford JA, Poldrack RA (2013) Complementary role of frontoparietal activity and cortical pattern similarity in successful episodic memory encoding. Cereb Cortex 23:1562-1571. CrossRef Medline

Zeithamova D, Preston AR (2010) Flexible memories: differential roles for medial temporal lobe and prefrontal cortex in cross-episode binding. J Neurosci 30:14676-14684. CrossRef Medline 